\title{
Stereotactic body radiotherapy as the initial treatment for hepatocellular carcinoma with extensive inferior vena cava and atrium tumor thrombus
}

This article was published in the following Dove Press journal:

OncoTargets and Therapy

\begin{abstract}
Yongjie Shui
Xiaoping Zhu'

Jianjun $\mathrm{Wu}^{2}$

Tingbo Liang ${ }^{3}$

Qichun Wei ${ }^{1,4}$

'Department of Radiation Oncology, The Second Affiliated Hospital, Zhejiang University School of Medicine, Hangzhou 310009, People's Republic of China;

${ }^{2}$ Department of Radiology, The Second Affiliated Hospital, Zhejiang University School of Medicine, Hangzhou 310009, People's Republic of China; ${ }^{3}$ Department of Hepatobiliary and Pancreatic Surgery, The Second Affiliated Hospital, Zhejiang University School of Medicine, Hangzhou 310009, People's Republic of China; ${ }^{4}$ Ministry of Education Key Laboratory of Cancer Prevention and Intervention, Zhejiang University Cancer Institute, Hangzhou 310009, People's Republic of China
\end{abstract}

Correspondence: Qichun Wei Department of Radiation Oncology, The Second Affiliated Hospital, Zhejiang University School of Medicine, Jiefang

Road 88, Hangzhou 310009, People's Republic of China

Email Qichun_Wei@zju.edu.cn

\begin{abstract}
Hepatocellular carcinoma (HCC) with inferior vena cava (IVC) tumor thrombus rarely occurs, its prognosis is extremely poor. A 49-year-old locally advanced HCC male with tumor thrombus extension into the IVC and the right atrium was successfully treated with stereotactic body radiotherapy (SBRT). A radiation dose of $40 \mathrm{~Gy} / 5$ fractions was delivered to the thrombus in 5 days. Contrast-enhanced magnetic resonance imaging one month after SBRT demonstrated no thrombus in IVC and the right atrium, and the huge lesion on right lobe also shrank. No severe adverse effect, eg, pulmonary embolism, was encountered. After controlling the thrombus, this patient has the opportunity to receive subsequent transarterial chemoembolization procedure in time, and avoided disease progression outside the irradiation volume. We reported a HCC case with extensive IVC and atrium tumor thrombus successfully treated by SBRT, the effectiveness and potential severe adverse effects were discussed.
\end{abstract}

Keywords: hepatocellular carcinoma, inferior vena cava tumor thrombus, atrium tumor thrombus, stereotactic body radiotherapy

\section{Introduction}

Hepatocellular carcinoma (HCC) with inferior vena cava tumor thrombus (IVCTT) rarely occurs, with a frequency of $1.4 \%,{ }^{1}$ it is reported to be with extremely poor prognosis, only 3 months survival for untreated patients. ${ }^{2}$

There is no standard treatment modality for HCC patients with IVCTT. Sorafenib is usually recommended for patients with advanced $\mathrm{HCC}$, with an expected median survival benefit of nearly 3 months. ${ }^{3,4}$ However, the efficacy of sorafenib in HCC patients with tumor thrombus is controversial. ${ }^{5}$ In a recently reported randomized Phase III trial, lenvatinib is non-inferior to sorafenib for overall survival, though with significantly improved progression-free survival, time to progression, and objective response. ${ }^{6}$ Patients with bile duct and main portal trunk invasion were excluded from the study, IVCTT cases might not be included. Transarterial chemoembolization (TACE) is the most commonly used procedure for unresectable HCC. However, it has relatively poor efficacy in resolving tumor thrombi, ${ }^{7,8}$ partly owing to the frequent arteriovenous shunts and extrahepatic collateral artery supply to the IVCTT. ${ }^{9}$ Local ablative therapies, such as radiofrequency ablation and microwave ablation, are contraindicated in tumors with vascular invasion. ${ }^{10}$ Surgical resection may be an effective 
treatment for HCC with IVCTT, only few cases are suitable for surgery due to advanced tumors or coexisting poor liver function. $^{11,12}$ More effective local therapy is needed for advanced HCC patients with IVCTT.

Several studies and our previous report ${ }^{13-15}$ demonstrated that stereotactic body radiotherapy (SBRT) has achieved good clinical results for portal vein tumor thrombus (PVTT). Therefore, it was reasonable to postulate that SBRT may also be used in the management of IVCTT. Here, we describe the application of SBRT in a patient with advanced HCC accompanied by extensive inferior vena cava (IVC) and atrium tumor thrombus.

\section{Case report}

A 49-year-old male complained of distending pain in his right upper abdomen for 2 weeks. He also complained of nausea and fatigue that began shortly before the onset of abdominal pain. He had a contrast-enhanced computed tomography (CT) and magnetic resonance imaging (MRI) of the upper abdomen that revealed multiple liver tumors on segments II, III, IV of left lobe and a huge lesion $(12.4 \mathrm{~cm})$ on segment VIII of right lobe, with thrombus in the left hepatic vein, middle hepatic vein, and the IVC which extended to the right atrium (Figure 1). Imaging findings of hepatic cirrhosis, ascites, and splenomegaly were also evidenced. The patient had a history of hepatitis B, with a high level of hepatitis B virus (HBV) DNA copy number $\left(1.63 \times 10^{3}\right)$.

The patient's liver function tests demonstrated: ALT 329 $\mathrm{U} / \mathrm{L}$, AST $231 \mathrm{U} / \mathrm{L}$, total bilirubin $17.8 \mu \mathrm{mol} / \mathrm{L}$, albumin 37.6 $\mathrm{g} / \mathrm{L}, \mathrm{LDH} 313 \mathrm{U} / \mathrm{L}$, and an international normalized ratio of 1.14. Tumor markers were negative for Alpha-fetoprotein and CA 19-9. CEA was elevated $(9.0 \mathrm{ng} / \mathrm{mL})$. After multidisciplinary team (MDT) evaluation, the diagnosis of hepatic cancer was established with clinical and imaging evidence according to the Chinese standard (version 2017). ${ }^{16}$ The case was regarded as unresectable, and SBRT was recommended to shrink the tumor thrombus and preserve liver function, preventing further extension of thrombus and with the hope to pursue subsequent TACE.

The patient was immobilized in a stereotactic body frame with customized vacuum cushion (Karity, Guangzhou, China) and abdominal compression for control of respiratory motion. Four-dimensional contrast-enhanced computed tomography simulation (Light Speed RT, GE) was performed at $2.5 \mathrm{~mm}$ slice thickness. The gross tumor volume (GTV) was delineated as the tumor thrombus in the left hepatic vein, middle hepatic vein, IVC, and in the right atrium. Internal target volume (ITV) was defined as the volumetric sum of GTVs in the multiple phases. ITV margin was expanded by $3 \mathrm{~mm}$ to set the planning target volume (PTV). The volume of PTV was $262.31 \mathrm{~cm}^{3}$. The patient was treated under institutional review board guidelines of the Second Affiliated Hospital, Zhejiang University School of Medicine, with signed informed consent. Written informed consent for the publication of the case details and accompanying images was provided from the patient, institutional approval was not required. He received a radiation dose of $40 \mathrm{~Gy} / 5$ fractions administered over a week (Figure 1).

The patient was relieved of his pain in 1 week. Contrastenhanced MRI was done one month after SBRT, the thrombus in the left hepatic vein dramatically shrank, and no thrombus in the middle hepatic vein, IVC, and the right atrium be found. The ascites disappeared. Moreover, the huge lesion on segment VIII of right lobe shrank to $9.1 \mathrm{~cm}$ (Figure 2). The liver function was improved, ALT and AST level decreased to 67 and $46 \mathrm{U} / \mathrm{L}$, respectively. HBV DNA copy number came back to normal range after anti-virus therapy.

Another MDT meeting was held, and it was decided that TACE would be the best option for the patient at this period. We used TACE targeting at tumors on segments II, III, IV of left lobe and VIII of right lobe. The microcatheter was advanced into tumor feeding artery of each tumor. A total dose of pirarubicin (40 mg)-lipiodol (15 mL) emulsion, 5\%

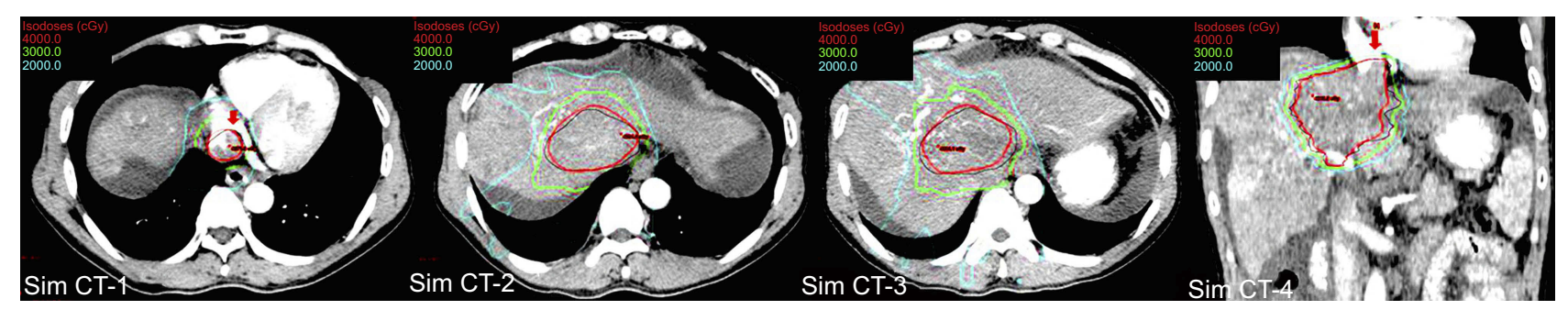

Figure I Images of the radiation treatment plan demonstrating the treatment volume (black), the 4,000 cGy isodose line (red), the 3,000 cGy isodose line (green), and the $2,000 \mathrm{cGy}$ isodose line (cyan). Tumor thrombus in the right atrium, inferior vena cava, and the hepatic vein were irradiated. The huge lesion on segment VIII of right lobe was not irradiated intentionally. The red solid arrow indicates inferior vena cava and tumor thrombus. 


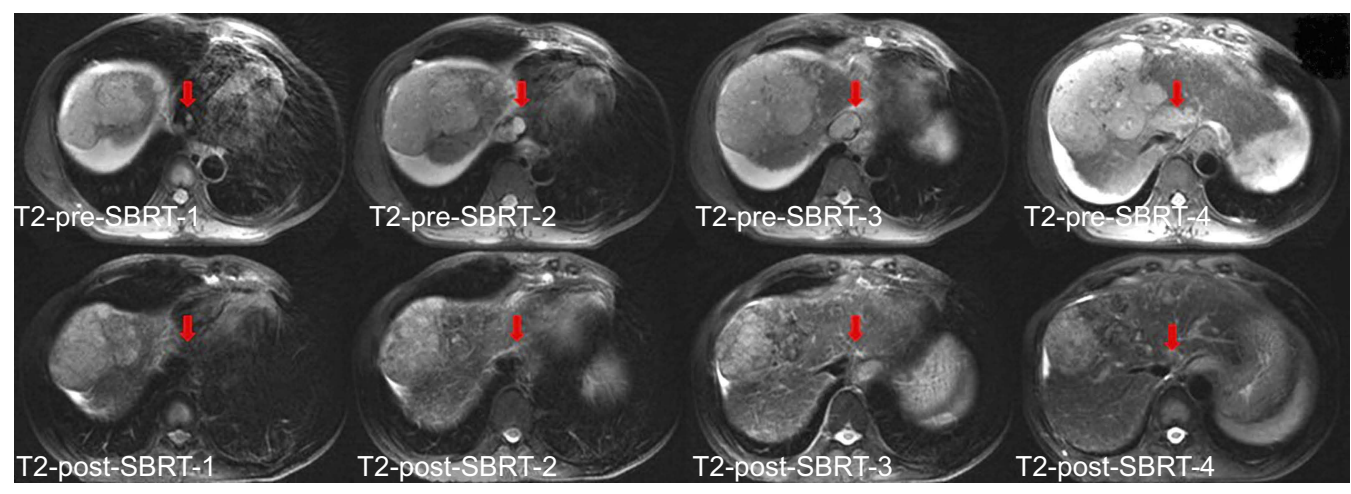

Figure 2 T2-weighted image. Upper row: pre-SBRT magnetic resonance imaging showing multiple liver tumors on segments II, III, IV of left lobe and a huge lesion (I2.4 cm) on segment VIII of right lobe, with thrombus in the left hepatic vein, middle hepatic vein, and the inferior vena cava which extended to the right atrium. Lower row: one month after SBRT, the thrombus in the inferior vena cava and the right atrium disappeared. The huge lesion on segment VIII of right lobe shrank to $9.1 \mathrm{~cm}$. The red solid arrow indicates inferior vena cava and tumor thrombus.

Abbreviation: SBRT, stereotactic body radiotherapy.

bicarbonate $100 \mathrm{~mL}$, oxaliplatin $150 \mathrm{mg}$, irinotecan $100 \mathrm{mg}$, PVA microsphere (300-500 $\mu \mathrm{m}$ and 700-900 $\mu \mathrm{m})$, gelatin sponge particles $(710-1,000 \mu \mathrm{m})$, were alternatively injected until the blood flow stopped. Microcoil was used to prevent recanalization of the embolized tumor feeding arteries with diameters larger than $2 \mathrm{~mm}$. The patient tolerated TACE well. Thirty days after TACE, the patient had MRI examination, which demonstrated a $94 \%$ necrosis, leaving around $6 \%$ viable residue. Selective deposit of the lipiodol oil only on tumor was also confirmed by CT scan. His CEA was $6.8 \mathrm{ng} / \mathrm{mL}$, CA 19-9 and Alpha-fetoprotein values were normal. The patient remained in stable condition with Child-Pugh A after SBRT and TACE. The patient received two TACE with more than 6 weeks interval, and had been doing well for more than half a year. Ten months after SBRT, the patient was hospitalized with a 1-week history of obstructive jaundice, an extrahepatic biliary obstruction was found in the procedure of percutaneous transhepatic cholangiography and drainage (PTCD) (Figure 3). The patient died 11 months after SBRT.

\section{Discussion}

The overall rate of incidence of IVCTT in HCC is low, ${ }^{1}$ and available evidence for the most appropriate therapeutic strategies for IVCTT is extremely limited. Palliative treatment with sorafenib or other systemic agents might be offered for these patients, the prognosis is dismal. Although an advanced HCC case with multiple metastasis and IVCTT showed complete response to Sorafenib was also reported. ${ }^{17}$ For patients suitable for surgery, the survival time might be improved. Recently, Chen et al, ${ }^{18}$ reported a relatively large series of 74 IVCTT cases treated with liver resection, a median overall survival (mOS) of 14 months was achieved

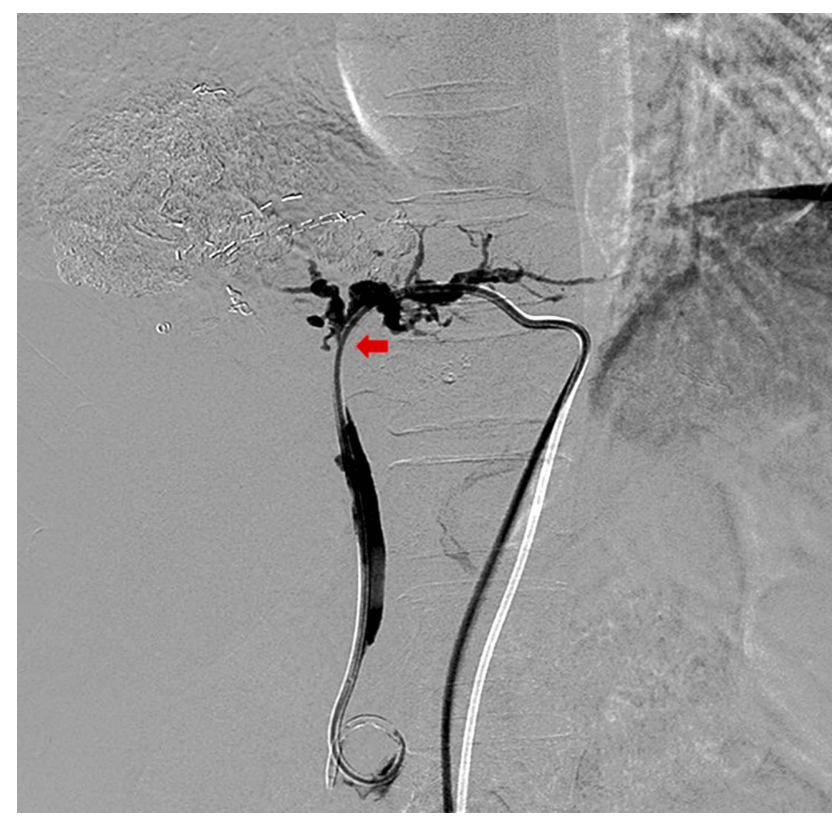

Figure 3 PTCD image shows extrahepatic bile duct stricture, while the intrahepatic bile ducts were dilatated. The red solid arrow indicates the location of the lesion.

Abbreviation: PTCD, percutaneous transhepatic cholangiography and drainage.

with acceptable complications. In the studies conducted by Wang et al, ${ }^{11}$ and Kokudo et al, ${ }^{19}$ mOS of 19 and 16.7 months was reported, respectively. These authors concluded that surgical resection can be considered in selected IVCTT patients with resectable primary tumor and sufficient hepatic reservoir. However, HCC patients with IVCTT are usually with advanced disease and poor liver function, only few cases are eligible for surgical resection. Although local ablative therapies are considered to be contraindication in tumors with vascular invasion, successful management of advanced HCC with IVCTT were reported in two cases, one by US- 
guided radiofrequency ablation, ${ }^{20}$ another by microwave ablation. $^{21}$ In addition to these notable cases managed by above-mentioned therapeutics, more effective local therapy is demanded.

In the present case report, we demonstrated tumor thrombus in IVC and atrium is sensitive to SBRT. Excellent therapeutic response was achieved which made it feasible for patient to endure further treatment. The efficacy of conventional fractionated radiotherapy for IVCTT has been reported in limited studies, ${ }^{22-24}$ but the therapeutic course is longer than 5 weeks. With the development of technologies of modern radiotherapy, SBRT is commonly used in most radiation oncology center. It delivers a large dose of radiation to a target, and the whole treatment course can be finished in few days resulting in less interference with other therapeutic measures. ${ }^{25} \mathrm{~A}$ recent review has discussed the role of external beam radiation therapy in the management of HCC, especially the potential indications of SBRT. ${ }^{26}$ Two articles concerning SBRT in the management of HCC patients with IVCTT were found. Xi et $\mathrm{al}^{27}$ reported eight HCC cases with IVCTT, a median dose of 36 Gy (range, 30-48 Gy) was delivered in six fractions over two weeks, the median survival was 9.2 months (95\% CI, 2.615.8 months). In the study conducted by Matsuo et al, ${ }^{14}$ six cases were HCC with IVCTT, 45-55 Gy was administered in 10-15 fractions, specific survival for IVCTT patients was not reported. The OS rate was significantly higher in the SBRT group than in the three-dimensional conformal radiation therapy (3D-CRT) group, and the authors suggested that SBRT may have the potential to be the standard RT technique for the treatment of PVTT/IVCTT. High biologically equivalent dose of radiation is engaged to kill tumor cells in the thrombus, which might facilitate intrathrombus resolution. The strategy seems practical to control the thrombosis by SBRT in a short therapeutic course, thus enable the patient to have the opportunity to receive subsequent TACE procedure in time, avoiding disease progression outside the irradiation volume.

It is noteworthy that the outfield huge lesion on segment VIII of right lobe also shrank after SBRT to the thrombus. Although the lesion was not irradiated intentionally, it actually got some dose (Figure 1). Another possible explanation might be an abscopal regression following SBRT, a rare phenomenon after radiotherapy as reported by Ohba et al. ${ }^{28}$

No pulmonary embolic event happened after SBRT in the present case. Theoretically, the risk of massive pulmonary embolism exists due to the possible dislodgement of the tumor thrombus in IVC and the atrium. SBRT delivers very high radiation dose to the IVCTT in few fractions which might increase the hazard of thrombus dropping out from the necrotized tumor into the IVC and the atrium. CRT in conventional dose fraction has been engaged in the treatment of IVCTT in previous reports. A pulmonary embolization rate of $5.5 \%(1 / 18)$ was documented in the study by Igaki et al. ${ }^{22}$ No pulmonary embolism or sudden death was detected in the patient series reported by Koo, et al. ${ }^{23}$ The authors speculate that the slow response of IVCTT might be crucial for the safety of after CRT treatment. In our case, rapid thrombus regression was implemented without pulmonary embolism, it seems feasible to apply SBRT for IVCTT treatment.

\section{Conclusions}

HCC with IVCTT rarely occurs, its prognosis is extremely poor. We reported a HCC case with extensive IVC and atrium tumor thrombus, the tumor thrombus was sensitive to SBRT and shrank dramatically. No severe adverse effect, eg, pulmonary embolism, was encountered. After controlling the thrombus, this patient got the opportunity to receive subsequent TACE procedure in time, and survived 11 months. The effectiveness and potential severe adverse effects were discussed.

\section{Disclosure}

The authors report no conflicts of interest in this work.

\section{References}

1. Kudo M, Izumi N, Ichida T, et al. Report of the 19th follow-up survey of primary liver cancer in Japan. Hepatol Res. 2016;46(5):372-390. doi:10.1111/hepr.12697

2. Nagasue N, Yukaya H, Hamada T, Hirose S, Kanashima R, Inokuchi $\mathrm{K}$. The natural history of hepatocellular carcinoma. A study of 100 untreated cases. Cancer. 1984;54(7):1461-1465.

3. Llovet JM, Ricci S, Mazzaferro V, et al. Sorafenib in advanced hepatocellular carcinoma. $N$ Engl J Med. 2008;359(4):378-390. doi:10.1056/NEJMoa0708857

4. Alejandro F, María R, Jordi B. Hepatocellular carcinoma. Lancet. 2018;391:1301-1314. doi:10.1016/S0140-6736(18)30010-2

5. Qi X, Guo X. Sorafenib for the treatment of hepatocellular carcinoma with portal vein tumour thrombosis: a systematic review of comparative studies. Prz Gastroenterol. 2015;10(3):142-147. doi:10.5114/ pg.2015.52470

6. Kudo M, Finn RS, Qin S, et al. Lenvatinib versus sorafenib in first-line treatment of patients with unresectable hepatocellular carcinoma: a randomised phase 3 non-inferiority trial. Lancet. 2018;391 (10126):1163-1173. doi:10.1016/S0140-6736(18)30207-1

7. Chung SM, Yoon CJ, Lee SS, et al. Treatment outcomes of transcatheter arterial chemoembolization for hepatocellular carcinoma that invades hepatic vein or inferior vena cava. Cardiovasc Intervent Radiol. 2014;37(6):1507-1515. doi:10.1007/s00270-014-0841-1

8. Yang QH, Zhang W, Liu QX, et al. TACE combined with implantation of irradiation stent versus TACE combine with bare stent for HCC complicated by IVCTT. Cardiovasc Intervent Radiol. 2016;39 (9):1280-1288. doi:10.1007/s00270-016-1372-8 
9. Lee IJ, Chung JW, Kim HC, et al. Extrahepatic collateral artery supply to the tumor thrombi of hepatocellular carcinoma invading inferior vena cava: the prevalence and determinant factors. $J$ Vasc Interv Radiol. 2009;20(1):22-29. doi:10.1016/j.jvir.2008.09.030

10. Nault JC, Sutter O, Nahon P, Ganne-Carrié N, Séror O. Percutaneous treatment of hepatocellular carcinoma: state of the art and innovations. $J$ Hepatol. 2018;68(4):783-797. doi:10.1016/j.jhep.2017.10.004

11. Wang Y, Yuan L, Ge RL, et al. Survival benefit of surgical treatment for hepatocellular carcinoma with inferior vena cava/right atrium tumor thrombus: results of a retrospective cohort study. Ann Surg Oncol. 2013;20(3):914-922. doi:10.1245/s10434-012-2646-2

12. Sakamoto K, Nagano H. Outcomes of surgery for hepatocellular carcinoma with tumor thrombus in the inferior vena cava or right atrium. Surg Today. 2018;48(9):819-824. doi:10.1007/s00595-0171619-2

13. Kang J, Nie Q, Du R, et al. Stereotactic body radiotherapy combined with transarterial chemoembolization for hepatocellular carcinoma with portal vein tumor thrombosis. Mol Clin Oncol. 2014;2(1):4350. doi:10.3892/mco.2013.196

14. Matsuo Y, Yoshida K, Nishimura H, et al. Efficacy of stereotactic body radiotherapy for hepatocellular carcinoma with portal vein tumor thrombosis/inferior vena cava tumor thrombosis: evaluation by comparison with conventional three-dimensional conformal radiotherapy. J Radiat Res. 2016;57(5):512-523. doi:10.1093/jrr/rrw028

15. Shui Y, Yu W, Ren X, et al. Stereotactic body radiotherapy based treatment for hepatocellular carcinoma with extensive portal vein tumor thrombosis. Radiat Oncol. 2018;13(1):188. doi:10.1186/ s13014-018-1136-5

16. Bureau of Medical Administration, National Health and Family Planning Commission of the People's Republic of China. Diagnosis, management, and treatment of hepatocellular carcinoma (V2017). Chin J Hepatol. 2017;25(12):886-895.

17. Simão A, Silva R, Correia L, et al. Advanced stage hepatocellular carcinoma with multiple metastasis and vascular thrombosis: a case of complete response to sorafenib. Acta Med Port. 2016;29(2):139142. doi:10.20344/amp.6799

18. Chen Z, Zhang X, Wang K, et al. Liver resection versus transcatheter arterial chemoembolization for the treatment of patients with hepatocellular carcinoma and hepatic vein or inferior vena cava tumor thrombus: a propensity score matching analysis. Hepatol Res. 2019;49:441-452. doi:10.1111/hepr.13297
19. Kokudo T, Hasegawa K, Yamamoto S, et al. Surgical treatment of hepatocellular carcinoma associated with hepatic vein tumor thrombosis. $J$ Hepatol. 2014;61(3):583-588. doi:10.1016/j. jhep.2014.04.032

20. Gatti P, Giorgio A, Ciracì E, et al. Hepatocellular carcinoma tumor thrombus entering the inferior vena cava treated with percutaneous RF ablation: a case report. J Ultrasound. 2019. Epub ahead of time. doi:10.1007/s40477-019-00361-w

21. Li W, Wang Y, Gao W, Zheng J. HCC with tumor thrombus entering the right atrium and inferior vena cava treated by percutaneous ablation. BMC Surg. 2017;17(1):21. doi:10.1186/s12893-017-0217-y

22. Igaki H, Nakagawa K, Shiraishi K, et al. Three-dimensional conformal radiotherapy for hepatocellular carcinoma with inferior vena cava invasion. Jpn J Clin Oncol. 2008;38(6):438-444. doi:10.1093/ jjco/hyn038

23. Koo JE, Kim JH, Lim YS, et al. Combination of transarterial chemoembolization and three-dimensional conformal radiotherapy for hepatocellular carcinoma with inferior vena cava tumor thrombus. Int J Radiat Oncol Biol Phys. 2010;78(1):180-187. doi:10.1016/j. ijrobp.2009.07.1730

24. Hou JZ, Zeng ZC, Zhang JY, et al. Influence of tumor thrombus location on the outcome of external-beam radiation therapy in advanced hepatocellular carcinoma with macrovascular invasion. Int $J$ Radiat Oncol Biol Phys. 2012;84(2):362-368. doi:10.1016/j. ijrobp.2011.12.024

25. Murray LJ, Dawson LA. Advances in stereotactic body radiation therapy for hepatocellular carcinoma. Semin Radiat Oncol. 2017;27 (3):247-255. doi:10.1016/j.semradonc.2017.02.002

26. Han B, Li C, Meng H, et al. Efficacy and safety of external-beam radiation therapy for hepatocellular carcinoma: an overview of current evidence according to the different target population. Biosci Trends. 2019;13(1):10-22. doi:10.5582/bst.2018.01261

27. Xi M, Zhang L, Zhao L, et al. Effectiveness of stereotactic body radiotherapy for hepatocellular carcinoma with portal vein and/or inferior vena cava tumor thrombosis. PLoS One. 2013;8(5):e63864. doi:10.1371/journal.pone.0063864

28. Ohba K, Omagari K, Nakamura T, et al. Abscopal regression of hepatocellular carcinoma after radiotherapy for bone metastasis. Gut. 1998;43(4):575-577. doi:10.1136/gut.43.4.575
OncoTargets and Therapy

\section{Publish your work in this journal}

OncoTargets and Therapy is an international, peer-reviewed, open access journal focusing on the pathological basis of all cancers, potential targets for therapy and treatment protocols employed to improve the management of cancer patients. The journal also focuses on the impact of management programs and new therapeutic agents and protocols on patient perspectives such as quality of life, adherence and satisfaction. The manuscript management system is completely online and includes a very quick and fair peer-review system, which is all easy to use. Visit http://www.dovepress.com/ testimonials.php to read real quotes from published authors. 